IJMMS 25:8 (2001) 525-532

PII. S0161171201003064

http://ijmms.hindawi.com

(c) Hindawi Publishing Corp.

\title{
SOME NEW INEQUALITIES FOR MEANS OF TWO ARGUMENTS
}

\section{JÓZSEF SÁNDOR and TIBERIU TRIF}

(Received 2 April 1999)

ABSTRACT. We prove certain new inequalities for special means of two arguments, including the identric, arithmetic, and geometric means.

2000 Mathematics Subject Classification. Primary 26D99, 65D32.

1. Introduction. The logarithmic and the identric mean of two positive real numbers $x$ and $y$ are defined by

$$
\begin{aligned}
& L=L(x, y):=\frac{y-x}{\log y-\log x}, \quad \text { if } x \neq y, \quad L(x, x)=x, \\
& I=I(x, y):=\frac{1}{e}\left(\frac{y^{y}}{x^{x}}\right)^{1 /(y-x)}, \quad \text { if } x \neq y, \quad I(x, x)=x,
\end{aligned}
$$

respectively.

Let $A=A(x, y):=(x+y) / 2$ and $G=G(x, y):=\sqrt{x y}$ denote the arithmetic and geometric means of $x$ and $y$, respectively. Many interesting results are known involving inequalities between these means. For a survey of results (cf. [1, 3, 4, 11, 13, 14]). Certain improvements are proved in [5, 7], while connections to other means are discussed, (cf. [6, 8, 9, 10, 15]). For identities involving various means we quote the papers $[6,12]$.

In $[5,8]$, the first author proved, among other relations, that

$$
\begin{gathered}
\left(A^{2} G\right)^{1 / 3}<I, \\
\left(U^{3} G\right)^{1 / 4}<I<\frac{U^{2}}{A},
\end{gathered}
$$

where

$$
U=U(x, y):=\left(\frac{8 A^{2}+G^{2}}{9}\right)^{1 / 2} .
$$

We note that a stronger inequality than (1.2) is (cf. [5])

$$
\frac{2 A+G}{3}<I
$$

but the interesting proof of (1.2), as well as the left-hand side of (1.3), is based on certain quadrature formulas (namely Simpson's and Newton's quadrature formula, respectively). As a corollary of (1.3) and (1.5), the double-inequality

$$
4 A^{2}+5 G^{2}<9 I^{2}<8 A^{2}+G^{2}
$$


can be derived (see [8]). Here and throughout the rest of the paper we assume that $x \neq y$.

The aim of this paper is twofold. First, by applying the method of quadrature formulas, we will obtain refinements of already known inequalities (e.g., of (1.2)). Second, by using certain identities on series expansions of the considered expressions, we will obtain the best possible inequalities in certain cases (e.g., for (1.6)).

\section{Main results}

THEOREM 2.1. If $x$ and $y$ are positive real numbers, then

$$
\begin{aligned}
\exp \left(\frac{(x-y)^{2}}{24 s^{2}}\right) & <\frac{A}{I}<\exp \left(\frac{(x-y)^{2}}{24 r^{2}}\right), \\
\exp \left(\frac{(x-y)^{2}}{12 s^{2}}\right) & <\frac{I}{G}<\exp \left(\frac{(x-y)^{2}}{12 r^{2}}\right), \\
\exp \left(\frac{(x-y)^{4}}{480 s^{4}}\right) & <\frac{I}{\left(A^{2} G\right)^{1 / 3}}<\exp \left(\frac{(x-y)^{4}}{480 r^{4}}\right), \\
\exp \left(\frac{(x-y)^{2}}{96 s^{2}}\right) & <\frac{\sqrt{3 A^{2}+G^{2}}}{2 I}<\exp \left(\frac{(x-y)^{2}}{96 r^{2}}\right),
\end{aligned}
$$

where $r=\min \{x, y\}$ and $s=\max \{x, y\}$.

Proof. Let $f:[0,1] \rightarrow \mathbb{R}$ be the function defined by $f(t)=\log (t x+(1-t) y)$. Since

$$
f^{\prime \prime}(t)=-\frac{(x-y)^{2}}{(t x+(1-t) y)^{2}}
$$

we have

$$
\begin{aligned}
& m_{2}:=\min \left\{-f^{\prime \prime}(t) \mid 0 \leq t \leq 1\right\}=\frac{(x-y)^{2}}{s^{2}}, \\
& M_{2}:=\max \left\{-f^{\prime \prime}(t) \mid 0 \leq t \leq 1\right\}=\frac{(x-y)^{2}}{r^{2}} .
\end{aligned}
$$

Applying the "composite midpoint rule" (cf. [2]) we get

$$
\int_{0}^{1} f(t) d t=\frac{1}{n} \sum_{i=1}^{n} f\left(\frac{2 i-1}{2 n}\right)+\frac{1}{24 n^{2}} f^{\prime \prime}\left(\xi_{n}\right), \quad\left(0<\xi_{n}<1\right) .
$$

Remarking that $I=\exp \left(\int_{0}^{1} \log (t x+(1-t) y) d t\right)$, relation (2.7) via (2.6) gives

$$
\exp \left(\frac{m_{2}}{24 n^{2}}\right)<\frac{\exp \left(1 / n \sum_{i=1}^{n} f((2 i-1) / 2 n)\right)}{I}<\exp \left(\frac{M_{2}}{24 n^{2}}\right) .
$$

Letting $n=1$, we get the double-inequality (2.1). For $n=2$, after a simple computation we deduce (2.4).

In order to prove (2.2), we apply the "composite trapezoidal rule" (see [2]):

$$
\int_{0}^{1} f(t) d t=\frac{1}{2}[f(0)+f(1)]-\frac{1}{12} f^{\prime \prime}(\eta), \quad(0<\eta<1) .
$$

As above, taking into account (2.6), relation (2.9) yields (2.2). 
Finally, (2.3) follows as application of the "composite Simpson rule" (see [2, 5]):

$$
\int_{0}^{1} f(t) d t=\frac{1}{6} f(0)+\frac{2}{3} f\left(\frac{1}{2}\right)+\frac{1}{6} f(1)-\frac{1}{2880} f^{(4)}(\zeta), \quad(0<\zeta<1) .
$$

We omit the details.

REMARKS. Inequality (2.8) is a common generalization of (2.1) and (2.4). The lefthand side of (2.3) is a refinement of (1.2), while the left-hand side of (2.4) implies the inequality

$$
4 I^{2}<3 A^{2}+G^{2},
$$

which slightly improves the right-side of (1.6). However, the best inequality of this type will be obtained by other methods.

In [6] the following identities are proved:

$$
\begin{aligned}
& \log \frac{I}{G}=\sum_{k=1}^{\infty} \frac{1}{2 k+1} z^{2 k}, \\
& \log \frac{A}{G}=\sum_{k=1}^{\infty} \frac{1}{2 k} z^{2 k}, \\
& \log \frac{I}{G}=\frac{A}{L}-1,
\end{aligned}
$$

where $z=(x-y) /(x+y)$.

Relation (2.14) is due to H.-J. Seiffert [11]. With the aid of these and similar identities, strong inequalities can be deduced. We first state the following.

THEOREM 2.2. The following inequalities are satisfied:

$$
\begin{aligned}
& \exp \left(\frac{1}{6}\left(\frac{x-y}{x+y}\right)^{2}\right)<\frac{A}{I}<\exp \left(\frac{(x-y)^{2}}{24 x y}\right), \\
& \exp \left(\frac{1}{3}\left(\frac{x-y}{x+y}\right)^{2}\right)<\frac{I}{G}<\exp \left(\frac{(x-y)^{2}}{12 x y}\right), \\
& \exp \left(\frac{1}{30}\left(\frac{x-y}{x+y}\right)^{4}\right)<\frac{I}{\left(A^{2} G\right)^{1 / 3}}<\exp \left(\frac{(x-y)^{4}}{120 x y(x+y)^{2}}\right) .
\end{aligned}
$$

Proof. We note that (2.16) appears in [6], while the left-hand side of (2.15) has been considered in [12]. We give here a unitary proof for (2.15), (2.16), and (2.17), which in fact shows that much stronger approximations may be deduced, if we want.

We assume that $x>y$, that is, $0<z<1$. Taking into account that

$$
\frac{z^{2}}{3}<\sum_{k=1}^{\infty} \frac{1}{2 k+1} z^{2 k}<\frac{z^{2}}{3}\left(1+z^{2}+z^{4}+\cdots\right)=\frac{z^{2}}{3\left(1-z^{2}\right)},
$$

from (2.12) we obtain the double-inequality (2.16).

On the other hand, (2.12) and (2.13) yield

$$
\log \frac{A}{I}=\sum_{k=1}^{\infty} \frac{1}{2 k(2 k+1)} z^{2 k} .
$$


Since

$$
\frac{z^{2}}{6}<\sum_{k=1}^{\infty} \frac{1}{2 k(2 k+1)} z^{2 k}<\frac{z^{2}}{6}\left(1+z^{2}+z^{4}+\cdots\right)=\frac{z^{2}}{6\left(1-z^{2}\right)},
$$

via (2.19) we get at once (2.15).

To prove (2.17), let us remark that from (2.12) and (2.19) we have

$$
\frac{I}{A^{2 / 3} G^{1 / 3}}=\exp \left(\sum_{k=2}^{\infty} \frac{k-1}{3 k(2 k+1)} z^{2 k}\right) \text {. }
$$

Since

$$
\frac{k-1}{3 k(2 k+1)} \leq \frac{1}{30} \quad \text { for all integers } k \geq 2,
$$

from (2.21) we get as above (2.17).

REMARKS. Inequalities (2.15), (2.16), and (2.17) improve (2.1), (2.2), and (2.3). From (2.14), taking account of (2.16), one can deduce that

$$
\frac{4\left(x^{2}+x y+y^{2}\right)}{3(x+y)^{2}}<\frac{A}{L}<\frac{x^{2}+10 x y+y^{2}}{12 x y} .
$$

In [4] it is proved that

$$
\log \frac{I}{L}>1-\frac{G}{L}
$$

Inequality (2.24) enabled the first author to obtain many refinements of known results (see [7]).

If one uses the estimations

$$
\frac{z^{2}}{6}+\frac{z^{4}}{20}<\sum_{k=1}^{\infty} \frac{1}{2 k(2 k+1)} z^{2 k}<\frac{z^{2}}{6}+\frac{z^{4}}{20}\left(1+z^{2}+z^{4}+\cdots\right)=\frac{z^{2}}{6}+\frac{z^{4}}{20\left(1-z^{2}\right)},
$$

as well as

$$
\frac{z^{2}}{3}+\frac{z^{4}}{5}<\sum_{k=1}^{\infty} \frac{1}{2 k+1} z^{2 k}<\frac{z^{2}}{3}+\frac{z^{4}}{5}\left(1+z^{2}+z^{4}+\cdots\right)=\frac{z^{2}}{3}+\frac{z^{4}}{5\left(1-z^{2}\right)},
$$

one could deduce the following inequalities:

$$
\begin{aligned}
& \exp \left(\frac{1}{6}\left(\frac{x-y}{x+y}\right)^{2}+\frac{1}{20}\left(\frac{x-y}{x+y}\right)^{4}\right)<\frac{A}{I}<\exp \left(\frac{1}{6}\left(\frac{x-y}{x+y}\right)^{2}+\frac{(x-y)^{4}}{80 x y(x+y)^{2}}\right) \\
& \exp \left(\frac{1}{3}\left(\frac{x-y}{x+y}\right)^{2}+\frac{1}{5}\left(\frac{x-y}{x+y}\right)^{4}\right)<\frac{I}{G}<\exp \left(\frac{1}{3}\left(\frac{x-y}{x+y}\right)^{2}+\frac{(x-y)^{4}}{20 x y(x+y)^{2}}\right)
\end{aligned}
$$

The next theorem provides a generalization of (2.17).

THEOREM 2.3. If $p$ and $q$ are positive real numbers with $2 q \geq p$, then

$$
\begin{aligned}
& \exp \left(\frac{2 q-p}{6}\left(\frac{x-y}{x+y}\right)^{2}+\frac{4 q-p}{20}\left(\frac{x-y}{x+y}\right)^{4}\right) \\
& <\frac{I^{p+q}}{A^{p} G^{q}}<\exp \left(\frac{2 q-p}{6}\left(\frac{x-y}{x+y}\right)^{2}+\frac{4 q-p}{80} \frac{(x-y)^{4}}{x y(x+y)^{2}}\right) .
\end{aligned}
$$


Proof. We assume that $x>y$, that is, $0<z<1$. From (2.12) and (2.19) we can deduce the following generalization of (2.21):

$$
\frac{I^{p+q}}{A^{p} G^{q}}=\exp \left(\sum_{k=1}^{\infty} \frac{2 k q-p}{2 k(2 k+1)} z^{2 k}\right)
$$

Since

$$
\frac{2 k q-p}{2 k(2 k+1)} \leq \frac{4 q-p}{20} \text { for all integers } k \geq 2
$$

we have

$$
\frac{2 q-p}{6} z^{2}+\frac{4 q-p}{20} z^{4}<\sum_{k=1}^{\infty} \frac{2 k q-p}{2 k(2 k+1)} z^{2 k}<\frac{2 q-p}{6} z^{2}+\frac{4 q-p}{20} \frac{z^{4}}{1-z^{2}} .
$$

The above estimation together with (2.29) yields (2.28).

REMARK 2.4. For $p=2 / 3$ and $q=1 / 3$, (2.28) gives (2.17), while for $p=q=1 / 2$ we get

$$
\begin{aligned}
\exp \left(\frac{1}{12}\right. & \left.\left(\frac{x-y}{x+y}\right)^{2}+\frac{3}{40}\left(\frac{x-y}{x+y}\right)^{4}\right) \\
& <\frac{I}{\sqrt{A G}}<\exp \left(\frac{1}{12}\left(\frac{x-y}{x+y}\right)^{2}+\frac{3}{160} \frac{(x-y)^{4}}{x y(x+y)^{2}}\right)
\end{aligned}
$$

THEOREM 2.5. If $x$ and $y$ are positive real numbers, then

$$
\exp \left(\frac{1}{45}\left(\frac{x-y}{x+y}\right)^{4}\right)<\frac{\sqrt{2 A^{2}+G^{2}}}{\sqrt{3} I}<\exp \left(\frac{1}{180} \frac{(x-y)^{4}}{x y(x+y)^{2}}\right) .
$$

Proof. Assume that $x>y$, that is, $0<z<1$. We prove first the following identity:

$$
\log \frac{\sqrt{\alpha A^{2}+G^{2}}}{\sqrt{\alpha+1} I}=\sum_{k=1}^{\infty} \frac{1}{2 k}\left(\frac{1}{2 k+1}-\frac{1}{(\alpha+1)^{k}}\right) z^{2 k},
$$

for all positive real numbers $\alpha$. Indeed, since

$$
\log \sqrt{\alpha A^{2}+G^{2}}=\log \sqrt{x y}+\log \sqrt{1+\frac{\alpha}{4}\left(\sqrt{\frac{x}{y}}+\sqrt{\frac{y}{x}}\right)^{2}},
$$

letting $z=(x-y) /(x+y)$ we obtain

$$
\begin{aligned}
\log \sqrt{\alpha A^{2}+G^{2}} & =\log G+\frac{1}{2} \log \left(1+\frac{\alpha}{1-z^{2}}\right) \\
& =\log G+\frac{1}{2} \log \left(1+\alpha-z^{2}\right)-\frac{1}{2} \log \left(1-z^{2}\right) .
\end{aligned}
$$

By the well-known formula

$$
\log (1-u)=-\sum_{k=1}^{\infty} \frac{u^{k}}{k}, \quad 0<u<1,
$$


we can deduce

$$
\log \left(1+\alpha-z^{2}\right)=\log (1+\alpha)-\sum_{k=1}^{\infty} \frac{z^{2 k}}{k(\alpha+1)^{k}}, \quad \log \left(1-z^{2}\right)=-\sum_{k=1}^{\infty} \frac{z^{2 k}}{k}
$$

Thus

$$
\log \frac{\sqrt{\alpha A^{2}+G^{2}}}{\sqrt{\alpha+1} G}=\sum_{k=1}^{\infty} \frac{1}{2 k}\left(1-\frac{1}{(\alpha+1)^{k}}\right) z^{2 k} .
$$

This identity combined with (2.12) ensures the validity of (2.34).

For $\alpha=2$, (2.34) yields

$$
\log \frac{\sqrt{2 A^{2}+G^{2}}}{\sqrt{3} I}=\sum_{k=2}^{\infty} \frac{1}{2 k}\left(\frac{1}{2 k+1}-\frac{1}{3^{k}}\right) z^{2 k} .
$$

Since

$$
\frac{1}{2 k}\left(\frac{1}{2 k+1}-\frac{1}{3^{k}}\right) \leq \frac{1}{45} \text { for all integers } k \geq 2,
$$

we have

$$
\frac{z^{4}}{45}<\sum_{k=2}^{\infty} \frac{1}{2 k}\left(\frac{1}{2 k+1}-\frac{1}{3^{k}}\right) z^{2 k}<\frac{z^{4}}{45\left(1-z^{2}\right)}
$$

This estimation together with (2.40) gives (2.33).

REMARKS. From (2.33) it follows that

$$
3 I^{2}<2 A^{2}+G^{2} .
$$

This inequality refines (2.11) and it is the best inequality of the type

$$
I^{2}<\frac{\alpha}{\alpha+1} A^{2}+\frac{1}{\alpha+1} G^{2} .
$$

Indeed, the function $f:] 0, \infty\left[\rightarrow \mathbb{R}\right.$ defined by $f(\alpha)=(\alpha /(\alpha+1)) A^{2}+(1 /(\alpha+1)) G^{2}$ is increasing because $A>G$. Taking into account (2.43) we get

$$
I^{2}<\frac{2}{3} A^{2}+\frac{1}{3} G^{2}<\frac{\alpha}{\alpha+1} A^{2}+\frac{1}{\alpha+1} G^{2}
$$

whenever $\alpha>2$. On the other hand, if $0<\alpha<2$, from (2.34) it follows that (2.44) cannot be true for all positive real numbers $x \neq y$.

The fact that (2.43) is the best inequality of the type (2.44) can be proved also by elementary methods, without resorting to series expansion (2.12). Indeed, letting $t=$ $(1 / 2)(x / y-1)$, and assuming that $x>y$, it is easily seen that (2.44) is equivalent to

$$
0<2 t-(1+2 t) \log (1+2 t)+t \log \left(1+2 t+\frac{\alpha}{\alpha+1} t^{2}\right)
$$

whenever $t>0$. Let $\left.g_{\alpha}:\right] 0, \infty[\rightarrow \mathbb{R}$ be the function defined by

$$
g_{\alpha}(t)=2 t-(1+2 t) \log (1+2 t)+t \log \left(1+2 t+\frac{\alpha}{\alpha+1} t^{2}\right) .
$$


We set, for convenience, $g_{2}:=g$. Easy computations give

$$
\begin{aligned}
& g^{\prime}(t)=\frac{2 t+(4 / 3) t^{2}}{1+2 t+(2 / 3) t^{2}}+\log \left(1+2 t+\frac{2}{3} t^{2}\right)-2 \log (1+2 t), \\
& \mathfrak{g}^{\prime \prime}(t)=\frac{8 t^{3}}{9(1+2 t)\left(1+2 t+(2 / 3) t^{2}\right)^{2}} .
\end{aligned}
$$

Since $g^{\prime \prime}(t)>0$ for all $t>0, g^{\prime}$ must be increasing. Therefore, $g^{\prime}(t)>0$ for $t>0$, because $g^{\prime}(0)=0$. Consequently $g$ is increasing, too. Hence $g(t)>0$ whenever $t>0$, because $g(0)=0$. This guarantees the validity of (2.46) for $\alpha=2$. Thus (2.43) is proved.

On the other hand, since

$$
\begin{gathered}
\log (1+2 t)=2 t-2 t^{2}+\frac{8 t^{3}}{3}+o\left(t^{3}\right) \\
\log \left(1+2 t+\frac{\alpha}{\alpha+1} t^{2}\right)=2 t+\frac{\alpha}{\alpha+1} t^{2}-\frac{1}{2}\left(2 t+\frac{\alpha}{\alpha+1} t^{2}\right)+o\left(t^{2}\right)
\end{gathered}
$$

it follows that

$$
g_{\alpha}(t)=\left(\frac{\alpha}{\alpha+1}-\frac{2}{3}\right) t^{3}+o\left(t^{3}\right)
$$

Therefore (2.46) cannot be true for all positive real numbers $t$ if $0<\alpha<2$.

\section{REFERENCES}

[1] H. Alzer, Ungleichungen für Mittelwerte [Inequalities for means], Arch. Math. (Basel) 47 (1986), no. 5, 422-426 (German). MR 88c:26022. Zbl 596.26015.

[2] G. Hämmerlin and K.-H. Hoffmann, Numerical Mathematics, Undergraduate Texts in Mathematics. Readings in Mathematics, Springer-Verlag, New York, 1991, translated from the German by Larry Schumaker. MR 92d:65001. Zbl 709.65001.

[3] E. Neuman, The weighted logarithmic mean, J. Math. Anal. Appl. 188 (1994), no. 3, 885900. MR 95k:26018. Zbl 823.33002.

[4] J. Sándor, On the identric and logarithmic means, Aequationes Math. 40 (1990), no. 2-3, 261-270. MR 91i:26028. Zbl 717.26014.

[5] _ _ A note on some inequalities for means, Arch. Math. (Basel) 56 (1991), no. 5, 471473. MR 92d:26028. Zbl 718.26017.

[6] _ On certain identities for means, Studia Univ. Babeş-Bolyai Math. 38 (1993), no. 4, 7-14. MR 97i:26030. Zbl 831.26013.

[7] _ On refinements of certain inequalities for means, Arch. Math. (Brno) 31 (1995), no. 4, 279-282. MR 97c:26028. Zbl 847.26015.

[8] _ Two inequalities for means, Int. J. Math. Math. Sci. 18 (1995), no. 3, 621-623. MR 96b:26030. Zbl 827.26016.

[9] _ On certain inequalities for means. II, J. Math. Anal. Appl. 199 (1996), no. 2, 629635. MR 97b:26023. Zbl 854.26013.

[10] J. Sándor and I. Raşa, Inequalities for certain means in two arguments, Nieuw Arch. Wisk. (4) 15 (1997), no. 1-2, 51-55. MR 98i:26026. Zbl 938.26011.

[11] H.-J. Seiffert, Comment to problem 1365, Math. Mag. 65 (1992), 365.

[12] U Ungleichungen für elementare Mittelwerte [Inequalities for elementary means], Arch. Math. (Basel) 64 (1995), no. 2, 129-131 (German). MR 95j:26026. Zbl 813.26009.

[13] K. B. Stolarsky, Generalizations of the logarithmic mean, Math. Mag. 48 (1975), 87-92. MR 50\#10186. Zbl 302.26003.

[14] _ The power and generalized logarithmic means, Amer. Math. Monthly 87 (1980), no. 7, 545-548. MR 82g:26029. Zbl 455.26008. 
[15] M. K. Vamanamurthy and M. Vuorinen, Inequalities for means, J. Math. Anal. Appl. 183 (1994), no. 1, 155-166. MR 95c:26021. Zbl 802.26009.

JÓzSEF SÁNDOR: FACUlTy of MATHEMATICS AND COMPUTER SCIENCE, BABEŞ-Bolyai UniVERSITY, 3400 CLUJ-NAPOCA, ROMANIA

E-mail address: jsandor@math.ubbc7uj.ro

Tiberiu Trif: Faculty of Mathematics and Computer SCIENCE, BabeŞ-Bolyai UniverSITY, 3400 CLUJ-NAPOCA, ROMANIA 


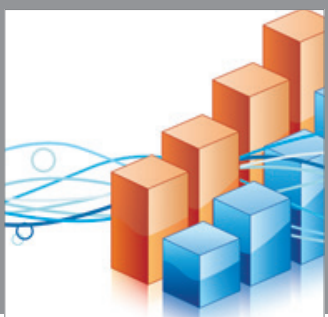

Advances in

Operations Research

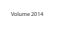

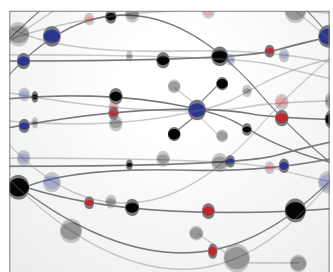

\section{The Scientific} World Journal
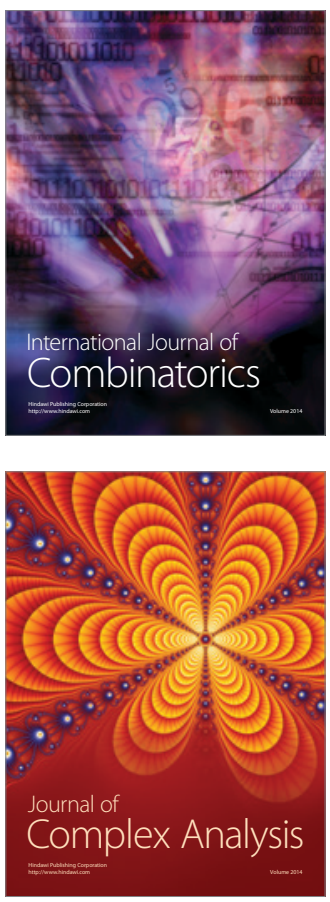

International Journal of

Mathematics and

Mathematical

Sciences
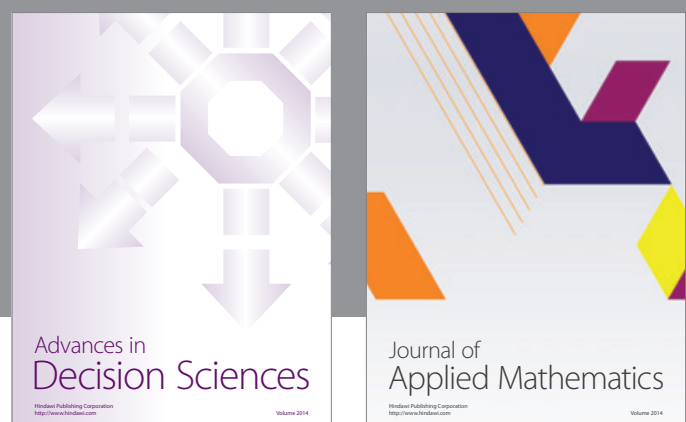

Journal of

Applied Mathematics
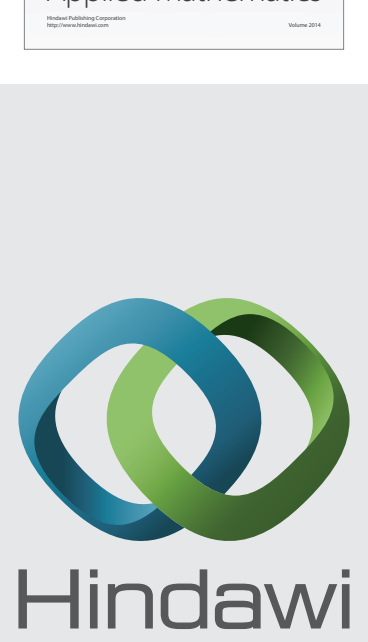

Submit your manuscripts at http://www.hindawi.com
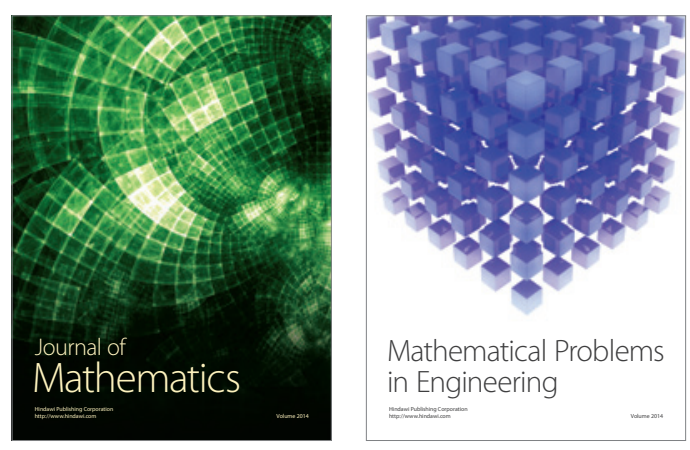

Mathematical Problems in Engineering
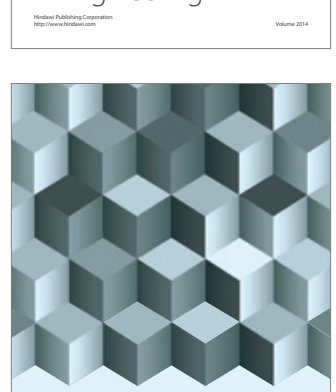

Journal of

Function Spaces
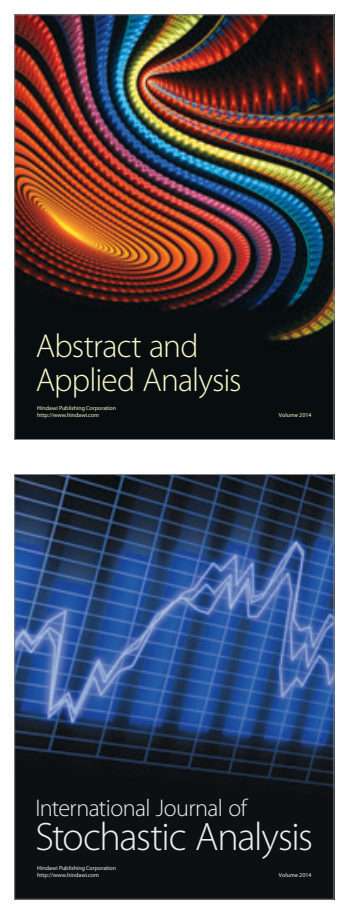

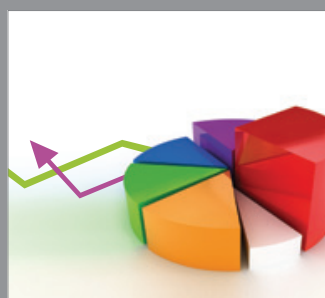

ournal of

Probability and Statistics

Promensencen
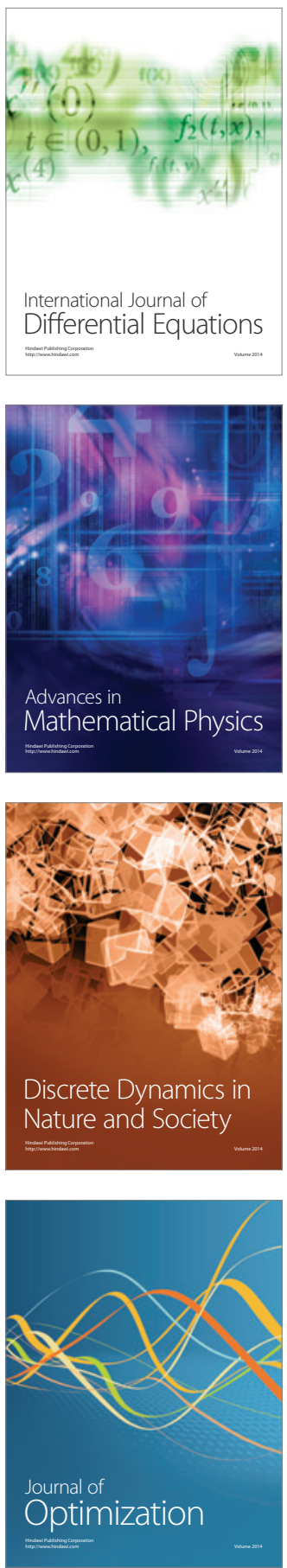